\title{
Aortic patch reconstruction of superior mesenteric and celiac axis in a deceased donor liver transplantation
}

\author{
N C Marasinghe ${ }^{1}$, R C Siriwardana ${ }^{2}$, S R E Wijesuriya ${ }^{3}$, C A H Liyanage ${ }^{3}$, H J De Silva ${ }^{4}$
}

Ceylon Medical Journal 2012; 57: 123

A liver was harvested for transplantation from a 35 year old patient who was brain dead following a road traffic accident. During the procedure it was noted that there was a replaced right hepatic artery arising from the superior mesenteric artery (SMA). The left hepatic artery was arising from the common hepatic artery. The graft was harvested preserving the celiac axis and the origin of the SMA in a common aortic cuff(Figures 1A and 1B). The SMA was divided $1 \mathrm{~cm}$ distal to the origin of the replaced right hepatic artery.

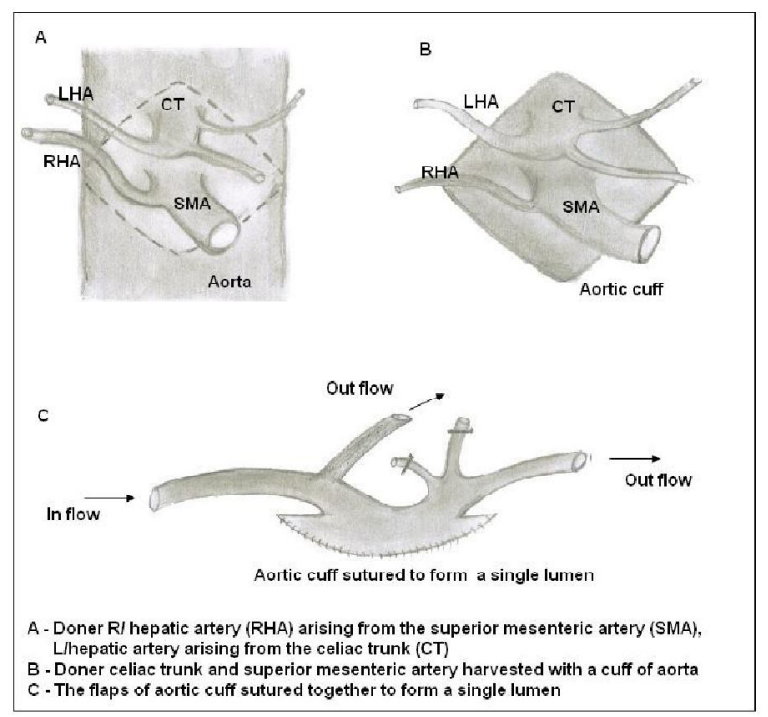

Figure 1. Schematic diagram of the aortic patch reconstruction.

During back table preparation it was decided to use the SMA as the inflow to the graft. An aortic patch at the origin of celiac axis and SMA was refashioned and reconstructed to create a common channel perfusing both left and right hepatic arteries (Figures $1 \mathrm{C}$ and 2). The recipient's proper hepatic artery bifurcation was used as the site of graft and recipient anastomosis. All anastomoses were performed with 7/0 polyglactin. Good arterial blood flow was re-established. The recipient was discharged from hospital on the eighth day after liver transplantation. A subsequent routine doppler scan showed normal pattern arterial blood flow.

A replaced right hepatic artery arising from the SMA occurs with a frequency of $12-20 \%$ [1]. These vessels usually pass lateral and behind the portal vein and enter the hepatoduodenal ligament posterolateral to the bile duct. When confronted with a variation such as this, the alternative is to anastomose the left and right hepatic arteries separately. But the technique we adopted was simple, technically easier and probably has a higher chance of vessel patency.

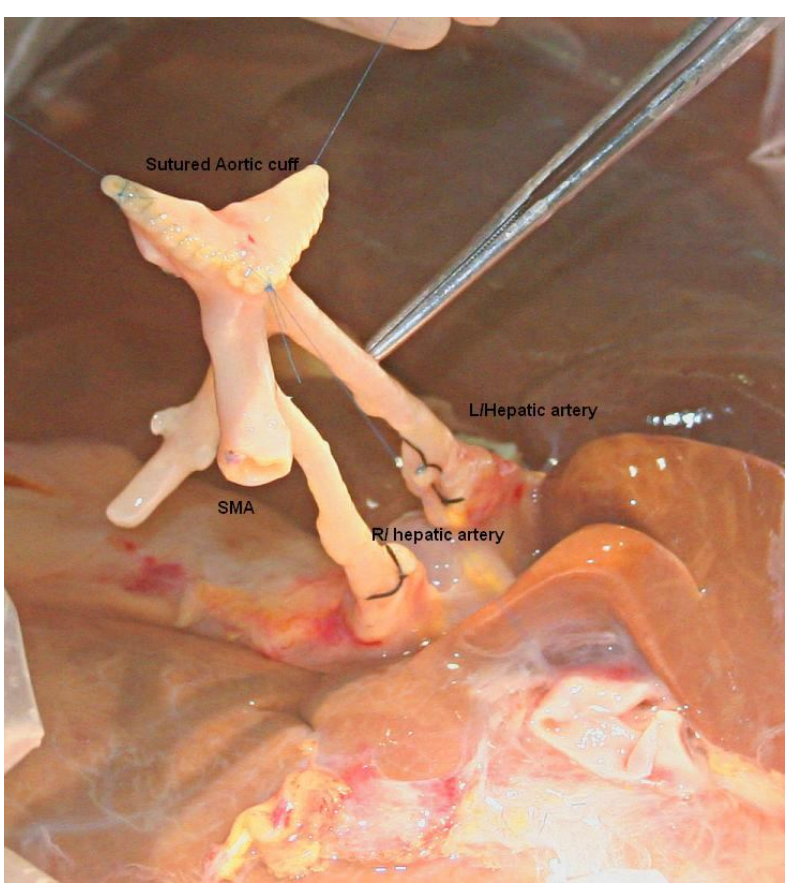

Figure 2. The reconstructed aortic patch.

\section{Reference}

1. Hiatt JR, Gabbay J, Busuttil RW. Surgical anatomy of the hepatic arteries in 1000 cases. Annals of Surgery 1994; 220: $50-2$.

${ }^{1}$ Surgical Unit and ${ }^{2}$ Gastro-Intestinal Surgery Unit, Colombo North Teaching Hospital, Ragama, Sri Lanka. Departments of ${ }^{3}$ Surgery and ${ }^{4}$ Medicine, Faculty of Medicine, University of Kelaniya, Sri Lanka.

Correspondence: RCS, e-mail: <rohansiriwardana@yahoo.com>. Received 19 August and revised version accepted 22 November 2011. Competing interests: none declared. 